\title{
Hydrogenation at low temperatures does not always lead to saturation: the case of $\mathrm{HNCO}$
}

\author{
J. A. Noble ${ }^{1}$, P. Theule ${ }^{1}$, E. Congiu ${ }^{2}$, F. Dulieu ${ }^{2}$, M. Bonnin ${ }^{2}$, A. Bassas ${ }^{1}$, F. Duvernay ${ }^{1}$, G. Danger ${ }^{1}$, and T. Chiavassa ${ }^{1}$ \\ ${ }^{1}$ Laboratoire Physique des Interactions Ioniques et Moléculaires, UMR 7345-CNRS, Aix-Marseille Université, \\ 13097 Marseille Cedex 20, France \\ e-mail: jennifer.noble@univ-amu.fr \\ 2 LERMA-LAMAp, Université de Cergy-Pontoise, Observatoire de Paris, ENS, UPMC, UMR 8112 du CNRS, 5 mail Gay Lussac, \\ 95000 Cergy Pontoise Cedex, France
}

Received 25 November 2014 / Accepted 30 January 2015

\begin{abstract}
Context. It is generally agreed that hydrogenation reactions dominate chemistry on grain surfaces in cold, dense molecular cores, saturating the molecules present in ice mantles.

Aims. We present a study of the low temperature reactivity of solid phase isocyanic acid (HNCO) with hydrogen atoms, with the aim of elucidating its reaction network.

Methods. Fourier transform infrared spectroscopy and mass spectrometry were employed to follow the evolution of pure HNCO ice during bombardment with $\mathrm{H}$ atoms. Both multilayer and monolayer regimes were investigated.

Results. The hydrogenation of HNCO does not produce detectable amounts of formamide $\left(\mathrm{NH}_{2} \mathrm{CHO}\right)$ as the major product. Experiments using deuterium reveal that deuteration of solid HNCO occurs rapidly, probably via cyclic reaction paths regenerating $\mathrm{HNCO}$. Chemical desorption during these reaction cycles leads to loss of $\mathrm{HNCO}$ from the surface.

Conclusions. It is unlikely that significant quantities of $\mathrm{NH}_{2} \mathrm{CHO}$ form from $\mathrm{HNCO}$. In dense regions, however, deuteration of $\mathrm{HNCO}$ will occur. HNCO and DNCO will be introduced into the gas phase, even at low temperatures, as a result of chemical desorption.
\end{abstract}

Key words. astrochemistry - ISM: molecules - molecular processes - molecular data

\section{Introduction}

Isocyanic acid, $\mathrm{HNCO}$, is the simplest molecule containing the four most abundant elements: hydrogen, carbon, nitrogen, and oxygen. It is an important interstellar molecule, with gas phase abundances of around $10^{-9}-10^{-8}$ with respect to $\mathrm{H}_{2}$ in molecular clouds, where it is believed to trace dense, cold gas (Jackson et al. 1984). Since its first detection in the Sgr B molecular cloud complex (Snyder \& Buhl 1972), $\mathrm{HNCO}$ has been detected in multiple environments, including hot cores (e.g. Helmich \& van Dishoeck 1997), high mass young stellar objects (Bisschop et al. 2007a), molecular outflows (Rodríguez-Fernández et al. 2010), comets (Lis et al. 1997), and other galaxies (Nguyen-Q-Rieu et al. 1991). It has been shown that HNCO is also a tracer of warm gas, and that its formation origins are likely to be predominantly in icy grain mantles (Bisschop et al. 2007a).

Isocyanic acid has not yet been observed in the solid state, but it is believed to be responsible for the formation of the abundant cyanate ion $\mathrm{OCN}^{-}$(e.g. Soifer et al. 1979; Demyk et al. 1998; Lowenthal et al. 2002; van Broekhuizen et al. 2005) via reaction of the acidic $\mathrm{HNCO}$ with bases such as $\mathrm{NH}_{3}$ (Raunier et al. 2003a; van Broekhuizen et al. 2004; Mispelaer et al. 2012) and $\mathrm{H}_{2} \mathrm{O}$ (Raunier et al. 2003b; Theule et al. 2011), or by the irradiation of ices with ultraviolet photons (Lacy et al. 1984) or protons (Moore et al. 1983). Moreover, its solid phase chemical reactions have been shown to give rise to isomerisation, with cyanic acid, HOCN, formed thermally in mixtures of HNCO and $\mathrm{H}_{2} \mathrm{O}$ (Theule et al. 2011).
The chemical network surrounding $\mathrm{HNCO}$ has not been fully studied experimentally. Early theoretical models assumed that HNCO formed only in the gas phase (e.g. Iglesias 1977), but more recent studies contend that $\mathrm{HNCO}$ forms on grain surfaces via the thermal reaction $\mathrm{NH}+\mathrm{CO}$ or by the hydrogenation of OCN (e.g. Garrod et al. 2008; Tideswell et al. 2010). Its presence in the gas phase is explained by the subsequent desorption of HNCO from grains, or by the destruction of larger species such as urea, $\left(\mathrm{NH}_{2}\right)_{2} \mathrm{CO}$. Abundances of $\mathrm{HNCO}$ are enhanced in shocked regions, and thus it is likely that sputtering or additional gas phase formation routes are active in post-shock gases (Zinchenko et al. 2000).

The abundance of solid HNCO is predicted to remain relatively low, at $\sim 10^{-4}$ with respect to $\mathrm{H}_{2} \mathrm{O}$, and thus is not detectable in infrared spectra. If the dominant formation route of HNCO (NH + CO) were efficient (Garrod et al. 2008), destruction routes would be required to explain this. As mentioned above, the formation of $\mathrm{OCN}^{-}$by reaction of $\mathrm{HNCO}$ with $\mathrm{H}_{2} \mathrm{O}$ and $\mathrm{NH}_{3}$ are favourable routes, the reactions having activation barriers of $26 \pm 2 \mathrm{~kJ} \mathrm{~mol}^{-1}(3130 \pm 240 \mathrm{~K}$; Theule et al. 2011) and $0.4 \pm 0.1 \mathrm{~kJ} \mathrm{~mol}^{-1}(48 \pm 12 \mathrm{~K}$; Mispelaer et al. 2012), respectively. Irradiation of $\mathrm{HNCO}$ with vacuum ultraviolet (VUV) radiation has been shown to produce formaldehyde $\left(\mathrm{H}_{2} \mathrm{CO}\right)$, formamide $\left(\mathrm{NH}_{2} \mathrm{CHO}\right)$, and urea $\left(\mathrm{H}_{2} \mathrm{NCONH}_{2}\right)$ (Raunier et al. 2004).

In dense molecular clouds the secondary photon field is weak and hydrogen atoms have a long residence time on grain surfaces because of the low temperature (Tielens \& Hagen 1982; Amiaud et al. 2007). Hydrogen atoms are mobile on the surface at $10 \mathrm{~K}$, 
and thus hydrogenation reactions dominate low temperature ice chemistry. Experimentally, there still remain many hydrogenation reactions to characterise, although studies have been carried out, particularly on simple molecules. The hydrogenation of $\mathrm{CO}$ to form $\mathrm{H}_{2} \mathrm{CO}$ and $\mathrm{CH}_{3} \mathrm{OH}$ has been extensively studied (e.g. Hiraoka et al. 1994; Watanabe \& Kouchi 2002; Fuchs et al. 2009), as have the reaction pathways to the formation of $\mathrm{H}_{2} \mathrm{O}$ from atomic oxygen (Hiraoka et al. 1998; Dulieu et al. 2010), molecular oxygen (Miyauchi et al. 2008; Ioppolo et al. 2008; Chaabouni et al. 2012), and ozone (Mokrane et al. 2009). The formation of $\mathrm{C}_{2} \mathrm{H}_{5} \mathrm{OH}, \mathrm{CH}_{4}, \mathrm{H}_{2} \mathrm{CO}$, and $\mathrm{CH}_{3} \mathrm{OH}$ from $\mathrm{CH}_{3} \mathrm{CHO}$ has been studied by Bisschop et al. (2007b), while the formation of $\mathrm{NH}_{2} \mathrm{OH}$ by the hydrogenation of $\mathrm{NO}$ (Congiu et al. 2012) and the formation of $\mathrm{CH}_{2} \mathrm{NH}$ and $\mathrm{CH}_{3} \mathrm{NH}_{2}$ from $\mathrm{HCN}$ (Theule et al. 2011) have recently been demonstrated.

Given that atomic hydrogen is present at fractional abundances of $\left[\mathrm{H}_{\mathrm{I}}\right] /\left[\mathrm{H}_{2}\right] \sim 10^{-3}$ in molecular clouds (Li \& Goldsmith 2003), hydrogenation is likely to dominate the destruction pathways of $\mathrm{HNCO}$ at low temperatures. Theoretical studies may suggest that the radical intermediates $\mathrm{HNCHO}$ or $\mathrm{NH}_{2} \mathrm{CO}$ form rapidly from $\mathrm{HNCO}$, and the stable molecule formamide forms on further hydrogenation of these intermediate species (Garrod et al. 2008), giving overall

$\mathrm{HNCO}+2 \mathrm{H} \rightarrow \mathrm{H}_{2} \mathrm{NCHO}$.

This work focuses on the reaction of $\mathrm{HNCO}$ with $\mathrm{H}$, which has not yet been examined experimentally. The experiments performed are introduced in Sect. 2, the results of these experiments are presented and discussed in Sect. 3, while the astrophysical implications of the results are considered in Sect. 4.

\section{Experimental}

Experiments were performed using two different experimental set-ups: RING, as described in Theule et al. (2011), and FORMOLISM, as described in Amiaud et al. (2006).

The RING set-up was used to perform multilayer, bulk ice experiments. Briefly, RING consists of a gold-plated copper surface within a high vacuum chamber (a few $10^{-9}$ mbar). Molecular species in the form of room temperature gas are dosed onto the gold surface $(15-300 \mathrm{~K})$ by spraying via an injection line. The infrared spectra of the molecular solids are recorded by means of Fourier transform reflection absorption infrared spectroscopy (FT-RAIRS) using a MCT detector in a Vertex 70 spectrometer. A typical spectrum has a $1 \mathrm{~cm}^{-1}$ resolution and is averaged over a few tens of interferograms.

The FORMOLISM set-up was used to perform experiments in the monolayer and sub-monolayer regime. The experimental set-up consists of an ultra high vacuum (UHV) chamber (base pressure $\sim 1 \times 10^{-10} \mathrm{mbar}$ ), containing a previously oxidised graphite HOPG sample (7-400 K, controlled by a closed-cycle He cryostat). Molecules are dosed onto the surface via two triply differentially pumped beam lines. Desorption of molecules from the surface is monitored using a quadrupole mass spectrometer (QMS, Hiden HAL-3F), positioned directly in front of the surface.

Isocyanic acid was prepared in the gas phase from cyanuric acid $(\mathrm{HNCO})_{3}$ via thermal decomposition of the commercially available trimer (Aldrich, $98 \%$ ) at $650^{\circ} \mathrm{C}$ under primary vacuum (Raunier et al. 2003b). Small quantities of $\mathrm{CO}_{2}$ and traces of CO are always present in the HNCO as a residual of the synthesis method.

In the multilayer experiments presented here, $\mathrm{HNCO}$ was dosed onto the surface held at $17 \mathrm{~K}$ via an injection line.

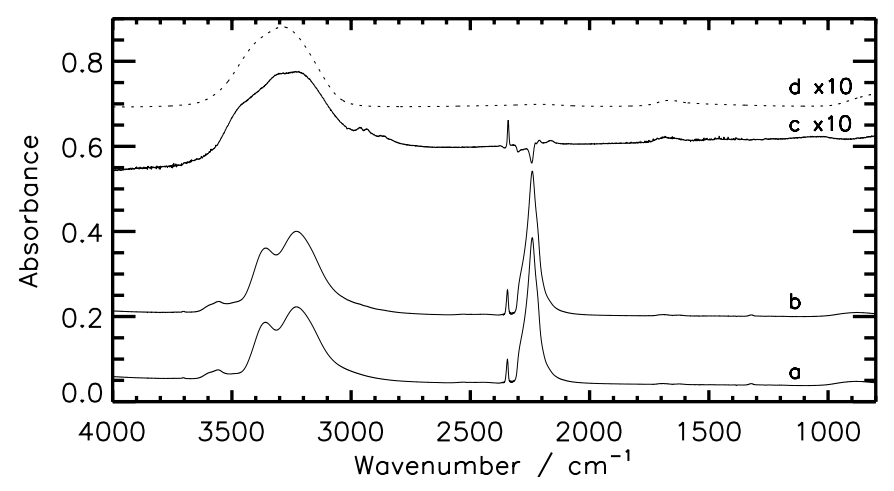

Fig. 1. Infrared absorption spectra of pure $\mathrm{HNCO}$ bombarded with $\mathrm{H}$. The spectra are as follows: a) pure HNCO deposited at $17 \mathrm{~K}$; b) HNCO bombarded with $\mathrm{H}$ atoms for $140 \mathrm{~min}$; and c) the difference spectrum of traces a and $b$. Trace d) is a reference spectrum discussed in full in the text.

The HNCO ice was bombarded with $\mathrm{H}$ atoms (at approximately $300 \mathrm{~K}$, with a flux of $\sim 10^{14} \mathrm{~cm}^{-2} \mathrm{~s}^{-1}$ ) produced in a molecular hydrogen plasma generated by a $2.45 \mathrm{GHz}$ microwave discharge. The plasma source and its calibration are fully detailed in Theule et al. (2011). The hydrogenation was monitored at regular time intervals using IR spectroscopy. In monolayer and sub-monolayer experiments, HNCO was dosed onto the surface held at $90 \mathrm{~K}$ via a molecular beam. This ensured that the deposited HNCO was not mixed with the byproduct $\mathrm{CO}_{2}$. The $\mathrm{HNCO}$ was cooled to $\sim 10 \mathrm{~K}$, then bombarded with $\mathrm{H}$ or $\mathrm{D}$ atoms (at approximately $300 \mathrm{~K}$, with a flux of $1 \pm 0.3 \times 10^{13} \mathrm{~cm}^{-2} \mathrm{~s}^{-1}$ ) produced in a hydrogen or deuterium plasma in the second molecular beam. After bombardment, the ices were probed using mass spectrometry during temperature programmed desorption (TPD) experiments.

\section{Results and discussion}

\subsection{The multilayer, bulk HNCO regime}

\subsection{1. $\mathrm{H}$ bombardment of HNCO}

The spectrum of pure multilayer $\mathrm{HNCO}$ deposited at $17 \mathrm{~K}$ is presented in Fig. 1, curve a. The molecular species HNCO is identified via its characteristic absorption bands, as listed in Table 1. The most intense bands are the $\mathrm{N}-\mathrm{H}$ stretching mode absorptions at 3554,3362 , and $3231 \mathrm{~cm}^{-1}$ and the $\mathrm{N}=\mathrm{C}=\mathrm{O}$ asymmetric stretching mode absorption at $2240 \mathrm{~cm}^{-1}$. A minor $\mathrm{CO}_{2}$ contamination is identified via the peak at $2344 \mathrm{~cm}^{-1}$.

The pure solid $\mathrm{HNCO}$ at $17 \mathrm{~K}$ was bombarded with $\mathrm{H}$ atoms for a total of $140 \mathrm{~min}$ (corresponding to a dose of $\sim 8 \times$ $10^{17} \mathrm{~cm}^{-2}$ ). The temperature of the deposition and $\mathrm{H}$ bombardment was chosen to be low enough to allow the $\mathrm{H}$ atoms to have a relatively long residency time on the surface, while being high enough to allow the $\mathrm{H}$ atoms to have a high mobility on the surface, to penetrate the bulk ice as deeply as possible, and to aid in overcoming any potential activation barrier to the hydrogenation of HNCO. The spectrum of the H-bombarded HNCO is presented in Fig. 1, curve b, while the difference spectrum of pure and H-bombarded HNCO is presented in Fig. 1, curve c. The positions of the absorption bands of the observed products are given in Table 1.

There are a number of crucial points to note with regard to the difference spectrum of H-bombarded HNCO (Fig. 1, curve c). First, in the wavelength region $2200-2300 \mathrm{~cm}^{-1}$ 
Table 1. Fundamental infrared band positions $\left(\mathrm{cm}^{-1}\right)$, for the species identified in the ice during the bombardment of $\mathrm{HNCO}^{a, b, c}$.

\begin{tabular}{lcccccc}
\hline \hline Species & $v \mathrm{~N}-\mathrm{H}$ & $v \mathrm{C}-\mathrm{H}$ & $v \mathrm{~N}=\mathrm{C}=\mathrm{O}_{\mathrm{as}}$ & $v \mathrm{C} \equiv \mathrm{O}$ & $v \mathrm{C}=\mathrm{O}$ & $\delta \mathrm{N}-\mathrm{H}_{\mathrm{s}}$ \\
\hline $\mathrm{HNCO}$ & $3554 / 3362 / 3231$ & - & 2240 & - & - & - \\
$\mathrm{NH}_{2} \mathrm{CHO}$ & $3313 / 3169^{c}$ & $2886^{c}$ & - & - & $1685^{b}$ & $1385^{c}$ \\
$\mathrm{OCN}^{-} \bullet$ & - & - & $2163^{a}$ & - & - & - \\
$\mathrm{CO}$ & - & - & - & $2136^{b}$ & - & - \\
$\mathrm{CO}_{2}$ & - & - & - & - & $2344^{a}$ & - \\
\hline
\end{tabular}

Notes. $v$ represents a stretching vibration, and $\delta$ a bending vibration. ${ }^{(a)}$ Positively identified in the H-bombarded HNCO ice. ${ }^{(b)}$ Tentatively identified in the H-bombarded $\mathrm{HNCO}$ ice. ${ }^{(c)}$ Not identified in the H-bombarded HNCO ice.

the peak absorption at $2240 \mathrm{~cm}^{-1}$ has diminished, indicative of a decrease of approximately 1 to 2 monolayers (of 580 total monolayers, calculated assuming a band strength of $7.8 \times 10^{-17} \mathrm{~cm} \mathrm{molec}^{-1}$ van Broekhuizen et al. 2004). Bands appearing at $\sim 3300 \mathrm{~cm}^{-1}$ and $1680 \mathrm{~cm}^{-1}$ are attributed to $\mathrm{H}_{2} \mathrm{O}$, and the band at $2342 \mathrm{~cm}^{-1}$ is attributed to $\mathrm{CO}_{2}$; both of these species are contaminants introduced via the $\mathrm{H}$ plasma, confirmed by blank experiments where the bare gold surface was bombarded. Figure 1, curve d is a pure $\mathrm{H}_{2} \mathrm{O}$ spectrum at $15 \mathrm{~K}$ fitted to the $1680 \mathrm{~cm}^{-1}$ band of the difference spectrum. Thus fitted, $\mathrm{H}_{2} \mathrm{O}$ accounts for most of the main feature in the difference spectrum (at $\sim 3300 \mathrm{~cm}^{-1}$ ) as well as the full $1680 \mathrm{~cm}^{-1}$ band. Remaining differences between the spectra of pure and $\mathrm{H}$-bombarded $\mathrm{HNCO}$ are relatively minor.

After inspection of the difference spectrum it appears that the expected hydrogenation product, methanamide (formamide, $\mathrm{NH}_{2} \mathrm{CHO}$ ), has not been produced in detectable quantities. Arguably the simplest potential product, formamide would be formed by the direct hydrogenation of $\mathrm{HNCO}$, as in Eq. (1). The left panel of Fig. 2 shows two alternative fits to the $1680 \mathrm{~cm}^{-1}$ feature (curve a); curve $b$ is the same scaled $\mathrm{H}_{2} \mathrm{O}$ spectrum as in Fig. 1, while curve $\mathrm{c}$ is a spectrum of pure $\mathrm{NH}_{2} \mathrm{CHO}$. The $\mathrm{H}_{2} \mathrm{O}$ ice feature is a qualitatively better fit to the band formed during the experiment, fully accounting for its width and red wing, while also accounting for the $3300 \mathrm{~cm}^{-1}$ feature as discussed above (see Fig. 1, curves c and d). However, traces of $\mathrm{NH}_{2} \mathrm{CHO}$ may have been formed during $\mathrm{H}$ bombardment and its absorption features are masked by the presence of $\mathrm{H}_{2} \mathrm{O}$. No other peaks attributable to $\mathrm{NH}_{2} \mathrm{CHO}$ were observed in the difference spectrum.

One further absorption feature in the difference spectrum can be analysed: a band appearing at $2163 \mathrm{~cm}^{-1}$, as illustrated in the right panel of Fig. 2 for $\mathrm{H}$ bombardment times of 20 (curve d), 80 (curve e), and 140 (curve f) minutes. The slight shoulder, centred at $\sim 2136 \mathrm{~cm}^{-1}$, can be attributed to $\mathrm{CO}$, which is a trace contaminant identified in the $\mathrm{H}$ plasma along with $\mathrm{H}_{2} \mathrm{O}$ and $\mathrm{CO}_{2}$. The $\mathrm{OCN}^{-}$ion is known to have an absorption band in the region of $2163 \mathrm{~cm}^{-1}$ (e.g. van Broekhuizen et al. 2004) and could form thermally (Theule et al. 2011) by reaction with the deposited $\mathrm{H}_{2} \mathrm{O}$ :

$\mathrm{HNCO}+\mathrm{H}_{2} \mathrm{O} \rightarrow \mathrm{OCN}^{-}+\mathrm{H}_{3} \mathrm{O}^{+}$.

The counterion $\mathrm{H}_{3} \mathrm{O}^{+}$was not observed in the $\mathrm{H}$-bombarded HNCO ice, but it should be noted that the band strength is very weak (Falk \& Giguère 1957), and much lower than that of $\mathrm{OCN}^{-}$ $\left(1.3 \times 10^{-16} \mathrm{~cm} \mathrm{ion}^{-1}\right.$ van Broekhuizen et al. 2004), so the absorption features would be vanishingly weak in our ice. If $\mathrm{OCN}^{-}$ is the species responsible for the band centred at $2163 \mathrm{~cm}^{-1}$, it is present in very small quantities: approximately 0.1 monolayers account for the peak produced after 140 min of $\mathrm{H}$ bombardment. However, given the quantity of $\mathrm{H}_{2} \mathrm{O}$ deposited over the course of the $\mathrm{H}$ bombardment, it is not unreasonable that $\mathrm{HNCO}$

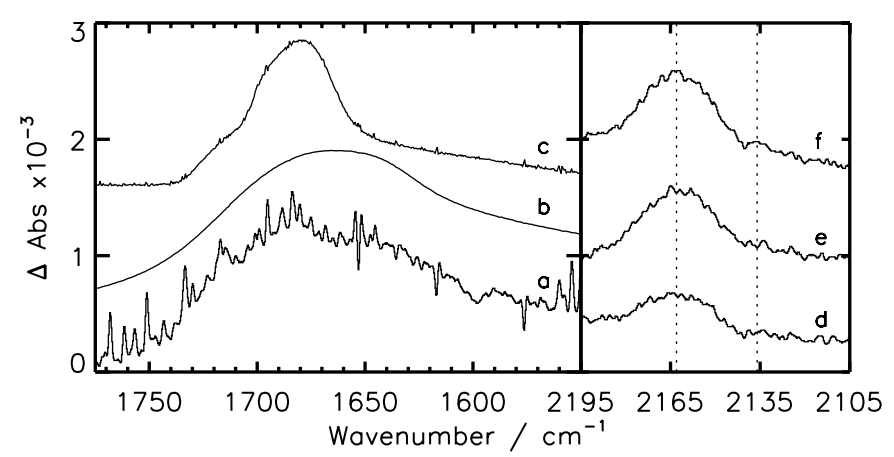

Fig. 2. Observed products of $\mathrm{H}+\mathrm{HNCO}$ in the multilayer regime. In the left panel, the spectra are as follows: a) the new band at $1680 \mathrm{~cm}^{-1}$ (magnification of the difference spectrum in Fig. 1c; b) a spectrum of pure $\mathrm{H}_{2} \mathrm{O}$, scaled to the band at $1680 \mathrm{~cm}^{-1}$ (magnification of Fig. 1d); and c) a spectrum of pure $\mathrm{NH}_{2} \mathrm{CHO}$, scaled to the band at $1680 \mathrm{~cm}^{-1}$. In the right panel, the spectra are the difference spectra of H-bombarded $\mathrm{HNCO}$ and pure HNCO are shown for bombardment times of d) $20 \mathrm{~min}$; e) $80 \mathrm{~min}$; and f) $140 \mathrm{~min}$ (magnification of the difference spectrum in Fig. 1c.

could react as in Eq. (2), as the rate of reaction was found to be $26 \mathrm{~kJ} \mathrm{~mol}^{-1}$ (Theule et al. 2011), which could be delivered to the ice surface by room temperature $\mathrm{H}$ atoms.

Another potential, but rather unlikely, explanation for the absorption feature is that the radical species NCO is formed by decomposition of $\mathrm{HNCO}$, probably by $\mathrm{H}$ abstraction:

$\mathrm{HNCO}+\mathrm{H} \rightarrow \mathrm{NCO}+\mathrm{H}_{2}$

The spontaneous decomposition of HNCO adsorbed on metal surfaces has been extensively reported in the surface science literature (e.g. Kiss \& Solymosi 1983; Celio et al. 1997; Jones \& Trenary 2008). The NCO radical formed by this decomposition is relatively stable, being observed on the surface at temperatures up to $600 \mathrm{~K}$ under UHV conditions (Celio et al. 1997). The position of the absorption band associated with the asymmetric stretching mode absorption of NCO is highly dependent on the environment, with a range of around $2145-2305 \mathrm{~cm}^{-1}$ (e.g. Németh et al. 2007).

One final comment to make about the multilayer reactivity of HNCO is that the VUV irradiation of pure HNCO has previously been shown to produce formaldehyde, formamide, and urea (Raunier et al. 2004). In this work, urea was not observed in the IR spectra after $\mathrm{H}$ bombardment of HNCO. As the $\mathrm{N}=\mathrm{C}$ bond must be broken to form urea, this route is unlikely. The only clear product of $\mathrm{H}$ bombardment of $\mathrm{HNCO}$ is the species responsible for the absorption band at $2163 \mathrm{~cm}^{-1}$; probably $\mathrm{OCN}^{-}$. The other absorption features remain ambiguous. 


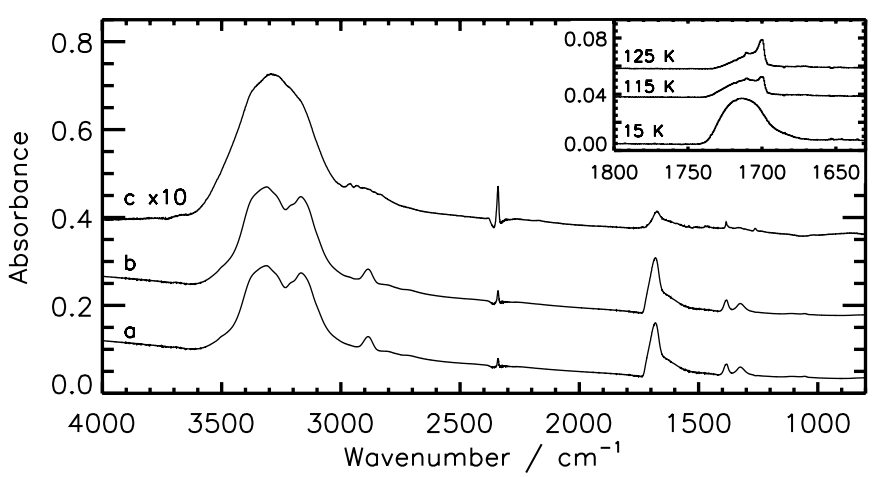

Fig. 3. Main panel: infrared absorption spectra of pure $\mathrm{NH}_{2} \mathrm{CHO}$ bombarded with $\mathrm{H}$ atoms. a) Pure $\mathrm{NH}_{2} \mathrm{CHO}$ deposited at $15 \mathrm{~K}$; b) $\mathrm{NH}_{2} \mathrm{CHO}$ after 190 min of $\mathrm{H}$ bombardment; c) the difference spectrum of a) and b). Inset: infrared spectra of $\mathrm{NH}_{2} \mathrm{CHO}$ deposited at $15 \mathrm{~K}$ then heated at $2 \mathrm{~K} \mathrm{~min}^{-1}$, taken at $15 \mathrm{~K}, 115 \mathrm{~K}$, and $125 \mathrm{~K}$.

\subsection{2. $\mathrm{H}$ bombardment of $\mathrm{NH}_{2} \mathrm{CHO}$}

As the results for the hydrogenation of HNCO were inconclusive, the reactivity of the expected product, formamide, was examined. The hydrogenation of formamide could lead to the production of aminomethanol according to

$\mathrm{NH}_{2} \mathrm{CHO}+2 \mathrm{H} \rightarrow \mathrm{NH}_{2} \mathrm{CH}_{2} \mathrm{OH}$.

The molecule $\mathrm{NH}_{2} \mathrm{CH}_{2} \mathrm{OH}$ is the most saturated form of the $\mathrm{NCO}$ moiety, so is the logical end-point for the hydrogenation chemistry of $\mathrm{HNCO}$.

Gas phase $\mathrm{NH}_{2} \mathrm{CHO}$ was dosed onto the surface held at $15 \mathrm{~K}$, then bombarded with $\mathrm{H}$ atoms for 190 min using the RING experimental set-up. The spectrum of pure $\mathrm{NH}_{2} \mathrm{CHO}$ at $15 \mathrm{~K}$ is presented in Fig. 3, curve a, with the H-bombarded sample spectrum as curve $b$. The difference spectrum of pure and $\mathrm{H}$-bombarded $\mathrm{NH}_{2} \mathrm{CHO}$ is presented in Fig. 3, curve c.

Pure formamide exhibits peaks at 3313, 3169, 2886, 1683, 1385 , and $1327 \mathrm{~cm}^{-1}$, as seen in Fig. 3, curve a. The $\mathrm{CO}_{2}$ molecule was present as a pollutant in the deposited $\mathrm{NH}_{2} \mathrm{CHO}$, as shown by the absorption feature in curve a (at $2341 \mathrm{~cm}^{-1}$ ), and in the $\mathrm{H}$ plasma, resulting in absorptions in curves $\mathrm{b}$ and $\mathrm{c}$. There is no clear decrease observed in the peak of the $v \mathrm{C}=\mathrm{O}$ absorption band at $1681 \mathrm{~cm}^{-1}$, and thus we conclude that $\mathrm{NH}_{2} \mathrm{CHO}$ has not reacted.

Similarly to the bombarded $\mathrm{HNCO}$, the difference spectrum of bombarded $\mathrm{NH}_{2} \mathrm{CHO}$ is dominated by absorption in the range $3600-3000 \mathrm{~cm}^{-1}$. As discussed above, this might have multiple sources, but a large contribution is due to $\mathrm{H}_{2} \mathrm{O}$. The four small peaks on the red wing between 2961 and $2859 \mathrm{~cm}^{-1}$ are again attributed to contamination of the $\mathrm{H}$ plasma due to primary vacuum. Such contamination is only observed because of the very long irradiation times. The peak observed in the difference spectrum at $1676 \mathrm{~cm}^{-1}$ is interpreted as further evidence of the deposition of $\mathrm{H}_{2} \mathrm{O}$, with a potential contribution from the crystallisation of the $\mathrm{NH}_{2} \mathrm{CHO}$ sample during $\mathrm{H}$ bombardment. We confirmed that, after heating a sample of pure $\mathrm{NH}_{2} \mathrm{CHO}$ at $2 \mathrm{~K} \mathrm{~min}^{-1}$, the crystallisation of $\mathrm{NH}_{2} \mathrm{CHO}$ results in the shifting of the $v \mathrm{C}=\mathrm{O}$ absorption band by $4-5 \mathrm{~cm}^{-1}$ towards the blue, as illustrated in the inset of Fig. 3. After H bombardment of $\mathrm{NH}_{2} \mathrm{CHO}$, no formation of $\mathrm{OCN}^{-}$was observed. We conclude that formamide is unreactive to $\mathrm{H}$ under our experimental conditions, and aminomethanol is not formed via this route.

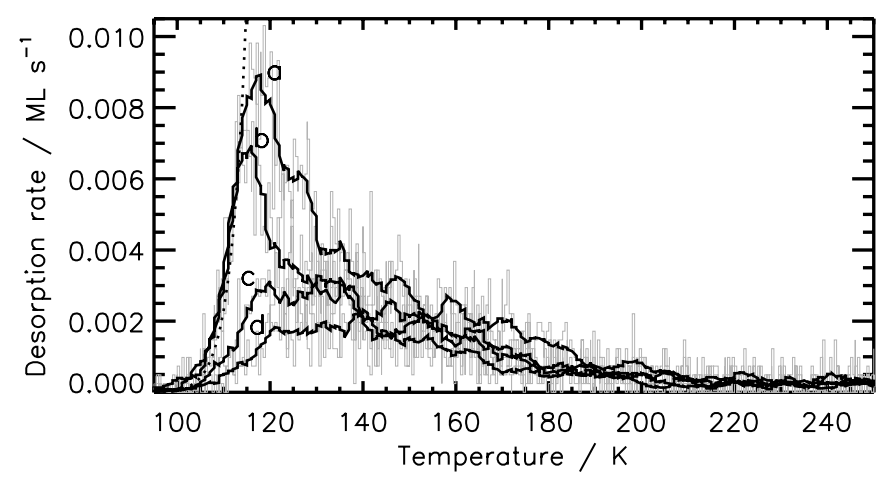

Fig. 4. Characterisation of the HNCO monolayer deposition. The TPD spectra $(\mathrm{m} / \mathrm{z}$ 43) for HNCO depositions of a) $1.5 \mathrm{ML}$; b) $1 \mathrm{ML}$; c) $0.75 \mathrm{ML}$; and d) $0.5 \mathrm{ML}$. The original data are plotted in grey, with a smoothed version overplotted in black. The Polanyi-Wigner fit to the leading edge of the 1.5 ML deposition (see Sect. 3.2.1) is plotted as a dotted line.

\subsection{The monolayer HNCO regime}

Because of the uncertainty in interpretation of our multilayer HNCO data, related to the low level contamination introduced by the $\mathrm{H}$ plasma of RING, we decided to perform experiments in the monolayer regime using FORMOLISM. Both experimental set-ups are described in Sect. 2, but the major advantage of FORMOLISM for this study is a base pressure of $\sim 10^{-8}$ mbar in the last stage of the molecular jets, which reduces the level of contamination of the $\mathrm{H}$ introduced into the chamber. Working in the monolayer regime allows us to bombard all deposited HNCO with $\mathrm{H}$, unlike the multilayer regime where hydrogen penetrates the first $\sim 1-3$ monolayers, and the signal of the bulk HNCO ice overwhelms that of product molecules in both the Fourier transform infrared (FTIR) and mass spectra. However, the monolayer regime also limits the signal-to-noise ratio of potential products observed using mass spectrometry and, in these experiments, we were unable to observe a clear signal for our HNCO reactant using FTIR spectroscopy.

\subsubsection{Desorption energy of $\mathrm{HNCO}$}

Using FORMOLISM, we performed experiments to determine the deposition conditions necessary to deposit $1 \mathrm{ML}$ of $\mathrm{HNCO}$ on the graphite surface; HNCO was deposited for a series of fixed times, and TPDs were performed after each deposition. The TPD spectra are shown in Fig. 4. The characteristic monolayer deposition time was determined by visual inspection of the leading edges of these spectra. All HNCO depositions were subsequently quantified by comparison with the identified monolayer deposition (Fig. 4, curve b). The low flux in the molecular beam allows us to deposit very reproducible quantities of molecular species. It takes almost $13 \mathrm{~min}$ to deposit $1 \mathrm{ML}$ of HNCO, so the deposition uncertainty on coverages of $0.5 \mathrm{ML}$ and $1 \mathrm{ML}$ (used in this work) is vanishingly small and the depositions are highly reproducible.

The multilayer desorption energy of HNCO was calculated via two different methods. The rate of desorption by unit surface, $r$, can be expressed by the Polanyi-Wigner equation (Redhead 1962; Carter 1962; King 1975), where the desorption rate constant $k_{\text {des }}$ is described in terms of an Arrhenius law,

$r=-\frac{\mathrm{d} N}{\mathrm{~d} T}=\frac{A}{\beta} \mathrm{e}^{-E_{\mathrm{des}} / R T} N^{n}$, 
where $A$ is the pre-exponential factor, $\beta=\frac{\mathrm{d} T}{\mathrm{~d} t}=12 \mathrm{~K} \mathrm{~min}^{-1}$ is the heating rate, $E_{\mathrm{des}}$ is the energy of desorption of a molecule from the surface $\left(\mathrm{J} \mathrm{mol}^{-1}\right), R$ is the gas constant $\left(\mathrm{J} \mathrm{K}^{-1} \mathrm{~mol}^{-1}\right)$, $T$ is the temperature of the surface $(\mathrm{K}), N$ is the number of adsorbed molecules on the surface (molecules $\mathrm{cm}^{-2}$ ), and $n$ is the order of the reaction. The units of $A$ depend on $n$ : molecules ${ }^{1-n} \mathrm{~cm}^{-2+2 n} \mathrm{~s}^{-1}$. When analysing the desorption of 1.5 ML of HNCO (Fig. 4, curve a), zeroth order desorption kinetics are assumed, as is standard practise for the multilayer desorption of bulk material, and therefore A has units of molecules $\mathrm{cm}^{-2} \mathrm{~s}^{-1}$.

By fixing the pre-exponential factor, $A$, at a value of $10^{28}$ molecules $\mathrm{cm}^{-2} \mathrm{~s}^{-1}$ (assuming that the lattice vibrational frequency of the solid is $10^{13} \mathrm{~s}^{-1}$ and the number of molecules in a monolayer is approximately $10^{15} \mathrm{~cm}^{-2}$ ), the desorption energy is calculated as the only free parameter in the fit of Eq. (5) to the leading edge of the experimental data (Collings et al. 2003). This fit is plotted as a dotted line in Fig. 4. Using this method, the calculated desorption energy was $E_{\mathrm{ads}, \mathrm{HNCO}}=32.9 \pm 1.7 \mathrm{~kJ} \mathrm{~mol}^{-1}$ $(3957 \pm 204 \mathrm{~K})$.

A second fitting method, proposed by Hasegawa et al. (1992), assumes that the pre-exponential factor in Eq. (5) is a function of $E_{\text {ads }}$ approximated by

$A=N_{\mathrm{ML}} \cdot v=N_{\mathrm{ML}} \sqrt{\frac{2 N_{\mathrm{ML}} E_{\mathrm{ads}}}{\pi^{2} M}}$,

where $M$ is the mass of the adsorbate molecule, and $N_{\mathrm{ML}} \sim$ $10^{15} \mathrm{~cm}^{-2}$. The advantage of this method is that the fit requires only one variable, $E_{\mathrm{des}}$, rather than assuming or fitting the preexponential factor and fitting $E_{\mathrm{des}}$ (Acharyya et al. 2007; Noble et al. 2012). Using the second method, the derived desorption energy was $E_{\text {ads }, \mathrm{HNCO}}=31.0 \pm 1.6 \mathrm{~kJ} \mathrm{~mol}^{-1}(3729 \pm 192 \mathrm{~K})$.

\subsection{2. $\mathrm{H}$ and $\mathrm{D}$ bombardment of $\mathrm{HNCO}$}

A series of experiments were performed on the $\mathrm{H}$ or $\mathrm{D}$ bombardment of monolayer and sub-monolayer quantities of solid $\mathrm{HNCO}$ at $10-15 \mathrm{~K}$. In this temperature range, $\mathrm{H}$ atoms do not form a bulk solid because of the rapid self recombination on the surface. A steady state, with an $\mathrm{H}_{2}$ coverage of $\sim 10 \%$ (Amiaud et al. 2007; Kristensen et al. 2011) due to desorption, is reached within the first ten seconds of $\mathrm{H}$ bombardment (Congiu et al. 2009).

Our initial experiments into the monolayer $\mathrm{H}$ or D bombardment of HNCO were carried out on short timescales. The results of 10 min (i.e. 1.7 ML) of H or D bombardment of $0.5 \mathrm{ML}$ HNCO are shown in Fig. 5. The left panel includes two curves: the TPD spectrum of $\mathrm{m} / \mathrm{z} 43$ (HNCO) for $0.5 \mathrm{ML}$ pure $\mathrm{HNCO}$ (curve a) and the corresponding spectrum after $10 \mathrm{~min}$ of $\mathrm{H}$ bombardment (curve b). The H-bombarded HNCO sample contains only 0.42 ML HNCO, which represents a $16 \%$ decrease compared to the pure HNCO sample. No m/z 45 (formamide) was observed to desorb during the TPD experiment, suggesting that no reaction occurred during $\mathrm{H}$ bombardment. However, the difference of $0.08 \mathrm{ML} \mathrm{HNCO}$ could be, at least partly, due to the low signal-to-noise ratio of the spectra (which is limited by the simultaneous measurement of multiple $\mathrm{m} / \mathrm{z}$ ).

The results of $\mathrm{D}$ bombardment, shown in the right panel of Fig. 5, are more revealing. After D bombardment we observe the species DNCO in our ice sample. In Fig. 5 the TPD spectrum of m/z 43 (HNCO) for $0.5 \mathrm{ML}$ pure $\mathrm{HNCO}$ is again plotted as curve a. The remaining curves, corresponding to $10 \mathrm{~min}$ of $\mathrm{D}$ bombardment (1.7 ML), are m/z 43 (HNCO, curve c) and m/z 44

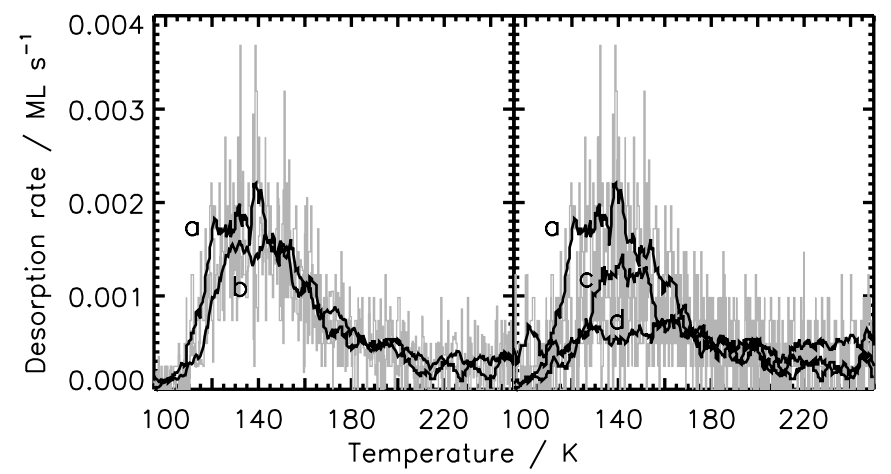

Fig. 5. H or D bombardment of HNCO. Left panel: TPDs of a) $0.5 \mathrm{ML}$ HNCO (m/z 43, HNCO), b) 0.5 ML HNCO + $10 \mathrm{~min} \mathrm{H} \mathrm{(m/z} \mathrm{43).} \mathrm{Right}$ panel: TPDs of a) $0.5 \mathrm{ML}$ HNCO (m/z 43, HNCO); c) $0.5 \mathrm{ML} \mathrm{HNCO} \mathrm{+}$ $10 \mathrm{~min} \mathrm{D} \mathrm{(m/z} \mathrm{43);} \mathrm{d)} \mathrm{as} \mathrm{for} \mathrm{spectrum} \mathrm{c),} \mathrm{but} \mathrm{for} \mathrm{m} / \mathrm{z} 44$ (DNCO). The original data are plotted in grey, with a smoothed version overplotted in black.

(DNCO, curve d), respectively. In this experiment, only 0.33 ML of HNCO remain after 10 min of D bombardment, representing a decrease of $34 \%$. Assuming that the mass spectrometer is equally sensitive to $\mathrm{HNCO}$ and DNCO, we calculate the quantity of DNCO on the surface after D bombardment to be $\sim 0.18 \mathrm{ML}$. Thus, the total quantity of HNCO and DNCO on the surface represents $0.51 \mathrm{ML}$, i.e. equivalent to the quantity of $\mathrm{HNCO}$ originally deposited. We will discuss the implications of the deuteration of HNCO in Sect. 3.2.4. No m/z 47 (NHDCDO, formamide) desorbed during the TPD, therefore we observe a conversion efficiency from HNCO to DNCO of approximately $100 \%$.

\subsubsection{The search for formamide}

In order to favour the formation of formamide, we performed an experiment in which we bombarded $1 \mathrm{ML}$ of HNCO with D for $150 \mathrm{~min}$ (25 ML), comparable to the multilayer bombardment times (see Sect. 3.1.1). The results of this experiment are presented in Fig. 6. A reference TPD of $1 \mathrm{ML}$ pure HNCO $(\mathrm{m} / \mathrm{z} 43)$ is shown in curve a. The HNCO $(\mathrm{m} / \mathrm{z} 43)$ and DNCO (m/z 44) on the surface after $25 \mathrm{ML}(150 \mathrm{~min})$ of $\mathrm{D}$ bombardment are shown in curves $\mathrm{b}$ and $\mathrm{c}$, accounting for $0.43 \mathrm{ML}$ and $0.22 \mathrm{ML}$, respectively. Thus, $0.35 \mathrm{ML}(35 \%)$ of the deposited HNCO is unaccounted for after D bombardment.

In order to determine the origin of the HNCO loss, we searched for the presence of formamide in the D-bombarded HNCO sample. The TPD method is typically sensitive to $\sim 0.01 \mathrm{ML}$ (Noble et al. 2011). In Fig. 7, curve a, we show the TPD spectrum of $1 \mathrm{ML}$ pure $\mathrm{HNCO}(\mathrm{m} / \mathrm{z} 43)$ as a reference. All other curves are $\mathrm{m} / \mathrm{z} 47$ (NHDCDO). Curve $b$ is from the same experiment as curve a, and indicates that there is no $\mathrm{m} / \mathrm{z} 47$ in the deposited HNCO. Curve $\mathrm{c}$ is a TPD performed after 120 min of D bombardment of the graphite surface, and shows that little or no $\mathrm{m} / \mathrm{z} 47$ is present in the atomic beam of D. Curve $d$ is the TPD of the HNCO sample bombarded with D for 150 min (see Fig. 6). There is no signal from m/z 47 after D bombardment of HNCO; we thus conclude that formamide is not formed at detectable levels (1\% of the deposited HNCO) during this experiment.

\subsubsection{Proposed reaction mechanism}

We conclude, based on the evidence from both multilayer and monolayer $\mathrm{H}$ and $\mathrm{D}$ bombardment of $\mathrm{HNCO}$, that the expected 


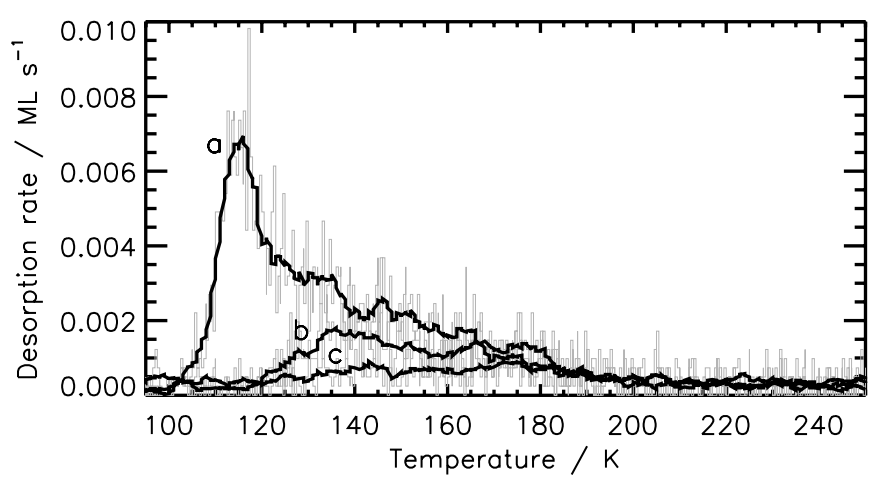

Fig. 6. Deuteration of HNCO. The spectra are a) reference TPD of $1 \mathrm{ML}$ HNCO (m/z 43); b) TPD after $25 \mathrm{ML}$ (150 min) of D bombardment of 1 ML HNCO (m/z 43, HNCO); c) as for spectrum b), but for $\mathrm{m} / \mathrm{z} 44$ (DNCO). The original data are plotted in grey, with a smoothed version overplotted in black.

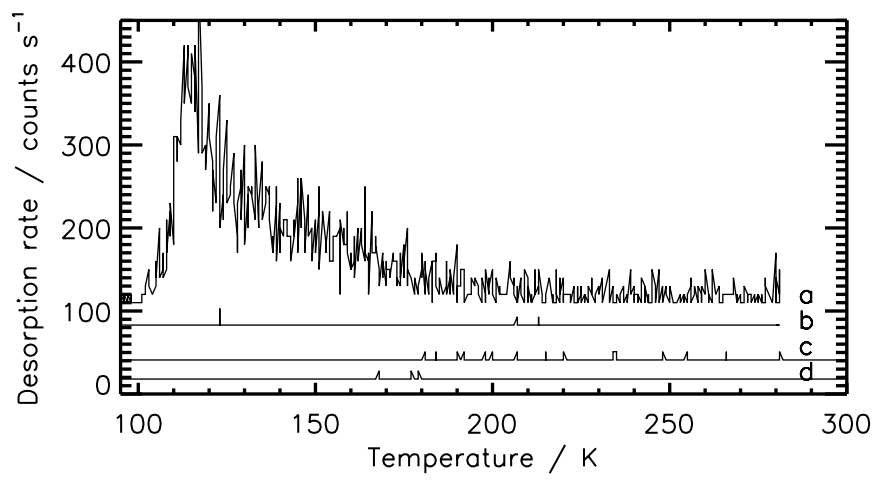

Fig. 7. Presence of $\mathrm{m} / \mathrm{z} 47$ in monolayer experiments. The spectra are a) $\mathrm{m} / \mathrm{z} 43$ desorbing during the TPD of $1 \mathrm{ML} \mathrm{HNCO}$; b) the corresponding desorption of $\mathrm{m} / \mathrm{z} 47$; c) $\mathrm{m} / \mathrm{z} 47$ after $120 \mathrm{~min}$ of D bombardment of the graphite surface; d) $\mathrm{m} / \mathrm{z} 47$ in an HNCO sample bombarded with $25 \mathrm{ML}(150 \mathrm{~min})$ of $\mathrm{D}$.

hydrogenation of $\mathrm{HNCO}$ to $\mathrm{NH}_{2} \mathrm{CHO}$ (Eq. (1)) does not readily occur and thus has a very high barrier. However, it is clear that D bombardment of HNCO produces DNCO and, additionally, that for longer bombardment times there is some loss of HNCO that is not accounted for by this DNCO formation. We provide a list of typical experiments, including the percentage loss of $\mathrm{HNCO}$, in Table 2. By comparing the loss of initial HNCO to the doses of $\mathrm{H}$ and $\mathrm{D}$, we see that there is a general trend in the data, whereby one molecule of HNCO is lost from the sample for approximately $1-1.5 \%$ of the incident $\mathrm{H}$ and D atoms. Although there was fluctuation in the absolute quantity of HNCO lost from the sample in our experiments, a loss was consistently seen for all $\mathrm{H}$ and $\mathrm{D}$ exposures greater than $\sim 2 \mathrm{ML}$. These losses are likely due to chemical desorption from the surface (Dulieu et al. 2013).

Chemical desorption is the stimulated desorption of reactant or product molecules due to an exothermic surface reaction. Recent experimental studies using FORMOLISM have highlighted the significance of the chemical desorption mechanism in the monolayer regime at low temperatures (Dulieu et al. 2013; Minissale et al. 2014; Noble et al. 2011). It has been shown that up to $90 \%$ of molecules (on a graphite surface) formed by radical-molecule or radical-radical surface reactions sublimate from the surface after formation because of the inability of the surface to quench the energy released during the reaction.
On ice surfaces, more astrophysically relevant for the study of molecules such as HNCO (which will not be present on bare grains), this number is typically much lower.

To account for both the formation of DNCO (after D bombardment) and the loss of HNCO from the surface (after both $\mathrm{H}$ and $\mathrm{D}$ bombardment), the chemistry is required to proceed via a cyclic pathway, such as the two proposed here:

$$
\begin{aligned}
& \mathrm{HNCO} \stackrel{+\mathrm{H}}{\longrightarrow} \mathrm{H}_{2} \mathrm{NCO} \stackrel{+\mathrm{H}}{\longrightarrow} \mathrm{HNCO}+\mathrm{H}_{2}, \\
& \mathrm{HNCO} \stackrel{+\mathrm{H}}{\longrightarrow} \mathrm{OCN}+\mathrm{H}_{2} \stackrel{+\mathrm{H}}{\longrightarrow} \mathrm{HNCO} .
\end{aligned}
$$

Cycle 7a corresponds to a classical hydrogenation pathway, i.e. $\mathrm{H}$ addition, but at the second step the branching ratio between the products $\mathrm{NH}_{2} \mathrm{CHO}$ (Eq. (1)) and $\mathrm{HNCO}$ is fully dominated by the reverse reaction to the initial $\mathrm{HNCO}$ population. Cycle $7 \mathrm{~b}$ corresponds to the abstraction of a hydrogen atom by the incident $\mathrm{H}$ atom. The reaction intermediates $\mathrm{H}_{2} \mathrm{NCO}$ and/or OCN are not expected to be observed in the TPDs as these radicals would either react with incident $\mathrm{H}$ atoms at a much faster rate than HNCO or, ultimately, recombine during the heating phase. It is also possible that, if cycle $7 \mathrm{~b}$ occurs, the second step could result in the isomerisation of HNCO to HOCN (as previously observed during the heating of mixtures of $\mathrm{HNCO}$ and $\mathrm{H}_{2} \mathrm{O}$ Theule et al. 2011). However, under current sub-monolayer experimental conditions it is not possible to differentiate between $\mathrm{HNCO}$ and HOCN, and no evidence of HOCN was observed in the multilayer experiments.

The results of our D bombardment experiments are critical to explaining the reaction mechanism. At a low dose of D (Table 2 and Fig. 5), we do not observe any loss of reactants or products from the surface, but deuteration appears to be very efficient. Around $34 \%$ of the initial HNCO $(0.17 \mathrm{ML})$ is transformed into DNCO. Of the total D atoms incident on the surface, approximately $10 \%$ have been included in the product. This represents a high efficiency, particularly when we consider that the reaction $\mathrm{D}+\mathrm{HNCO}$ is in competition with $\mathrm{D}+\mathrm{D}$. For comparison, the $\mathrm{CO}+\mathrm{H}$ system described by Fuchs et al. (2009) or Watanabe \& Kouchi (2002) uses only $1 \%$ of the incident $\mathrm{H}$ atoms in the formation of $\mathrm{H}_{2} \mathrm{CO}$ and $\mathrm{CH}_{3} \mathrm{OH}$. Although we perform our experiments under different conditions, we are able to place the reaction $\mathrm{HNCO}+\mathrm{H}$ between that of $\mathrm{H}+\mathrm{H}_{2} \mathrm{CO}$ (which is faster) and $\mathrm{H}+\mathrm{CO}$ (which is slower). It is clear that the barrier to the reaction $\mathrm{HNCO}+\mathrm{D}$ is not very high, and is probably in the region of $250-700 \mathrm{~K}$. A specific study investigating varying doses and surface temperatures would be required to calculate this value; this represents a large amount of work and is beyond the scope of this paper.

When we extend D bombardment to longer times, we observe a loss of material from the surface in addition to the formation of DNCO (Table 2 and Fig. 6). For an initial deposition of $1 \mathrm{ML}$ HNCO subjected to $25 \mathrm{ML}$ of D, we recover $43 \%$ of the $\mathrm{HNCO}$ and produce $22 \%$ DNCO i.e. a loss of $35 \%$ of the original material. Assuming that the proportion of $\mathrm{D}$ atoms reacting is the same as for short timescales $(\sim 10 \%)$, we approximate that $\mathrm{HNCO}+\mathrm{D}$ occurs $2.5 \times 10^{15} \mathrm{~cm}^{-2}$ times. The observed loss represents $3.5 \times 10^{14}$ molec $\mathrm{cm}^{-2}$, and we can make a rough estimation that the efficiency of chemical desorption is $\sim 14 \%$. Unlike in some previous studies (Dulieu et al. 2013), the relative efficiency of the desorption mechanism must be low, as only $0.35 \mathrm{ML}$ is lost from the surface over a bombardment period of $150 \mathrm{~min}$. This is equivalent to a desorption rate of $\sim 4 \times 10^{-5} \mathrm{ML} \mathrm{s}^{-1}$, and as such any desorbing molecules would not be observable using the mass spectrometer. The result 
Table 2. Results of experiments carried out on monolayer and sub-monolayer quantities of HNCO.

\begin{tabular}{lccccc}
\hline \hline $\begin{array}{l}\text { Initial HNCO } \\
(\mathrm{ML})\end{array}$ & Exposed to & $\begin{array}{c}\text { Temperature } \\
(\mathrm{K})\end{array}$ & $\begin{array}{c}\text { HNCO reduction } \\
(\%)\end{array}$ & $\begin{array}{c}\text { DNCO produced } \\
(\%)\end{array}$ & $\begin{array}{c}\text { Loss (to CD) } \\
(\%)\end{array}$ \\
\hline 0.5 & & 10 & $34 \pm 5$ & $36 \pm 5$ & $-2 \pm 5$ \\
1.0 & $1.7 \mathrm{ML} \mathrm{D}$ & 7 & $11 \pm 5$ & none & $11 \pm 5$ \\
1.0 & $10 \mathrm{ML} \mathrm{H}$ & 15 & $57 \pm 5$ & $22 \pm 5$ & $35 \pm 5$ \\
\hline
\end{tabular}

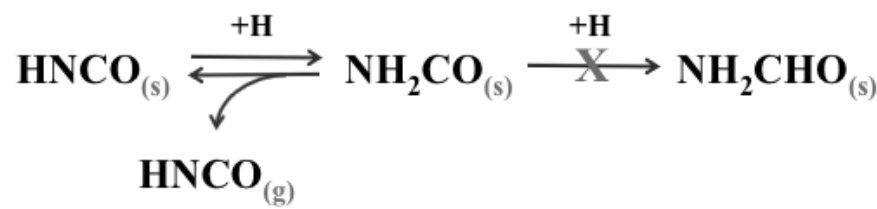

Fig. 8. Proposed reaction scheme for $\mathrm{HNCO}$ with $\mathrm{H}$.

indicates that the reaction driving the chemical desorption mechanism is not highly exothermic.

Each of the two reaction scenarios that we proposed above proceeds via two steps. The first step (H-abstraction or $\mathrm{H}$-addition) limits the kinetics of the overall reaction and is also less likely to provoke chemical desorption since less energy will be released than in the second radical-radical step. Thus most of the desorption should occur at the second reaction step. If the reactivity of both the HNCO and DNCO species was equivalent, we would assume that, with the longer exposures, over $90 \%$ of the initial HNCO should be transformed, which is not the case. The low deuteration yield could be due to the presence of a second reactant (DNCO) and/or steric limitation of the HNCO deposition. However, we propose that the most likely limiting factor is the stabilisation of the HNCO by its formation of dimers or larger polymers, preventing its reaction with $\mathrm{H}$. Experiments performed on $\mathrm{HNCO}$ in a $\mathrm{H}_{2} \mathrm{O}$ ice, or isolated in a low temperature matricial gas, would reduce the intermolecular bonding and may allow hydrogenation to occur. Because of the inability to observe desorbing $\mathrm{HNCO} / \mathrm{DNCO}$ (owing to the low rate of chemical desorption) and the hypothesised intermediates ( $\mathrm{OCN}$ and/or $\mathrm{H}_{2} \mathrm{NCO}$ ) by mass spectrometry, we are unable to determine whether one reaction cycle dominates the reactivity of $\mathrm{HNCO}+\mathrm{H}$. In order to differentiate between the abstraction and addition mechanisms, a full ab initio quantum mechanical calculation treatment including surface effects would be required.

By comparing our monolayer experiments with those in bulk ice, we confirm that formamide is not produced in detectable quantities after $\mathrm{H}$ bombardment of HNCO. It is also plausible that the inconsistency between the loss of $\mathrm{HNCO}(\sim 1-2 \mathrm{ML})$ and the only confirmed product $\mathrm{OCN}^{-} / \mathrm{OCN}(\sim 0.1 \mathrm{ML})$ in our bulk experiments can be explained by the proposed cyclic reactivity of HNCO combined with chemical desorption. The efficient $\mathrm{H} / \mathrm{D}$ cyclic substitution suggests that the N-H bond in $\mathrm{HNCO}$ is relatively weak, which strengthens the argument for a low level of HNCO decomposition into OCN. We show our proposed overall reaction scheme in Fig. 8.

\section{Astrophysical implications}

Our results contradict theoretical studies which proposed that the initial hydrogenation step to form either $\mathrm{HNCHO}$ or $\mathrm{NH}_{2} \mathrm{CO}$ is very important to $\mathrm{HNCO}$ chemistry and that subsequent hydrogenation leads to $\mathrm{NH}_{2} \mathrm{CHO}$ (e.g. Garrod et al. 2008; Tideswell et al. 2010). However, ab initio calculations determine an activation barrier of $1390 \mathrm{~K}$ to the first hydrogenation step (Nguyen et al. 1996). A recent experimental study of the formation of $\mathrm{HNCO}$ by addition of the radical $\mathrm{NH}$ to $\mathrm{CO}$ revealed no formation of $\mathrm{NH}_{2} \mathrm{CHO}$ after co-deposition of $\mathrm{N}, \mathrm{H}$, and $\mathrm{CO}$, despite formation of HNCO (Fedoseev et al. 2015).

The $\mathrm{NH}_{2} \mathrm{CHO}$ molecule has been tentatively identified in ices towards the objects W 33A (Schutte et al. 1999) and NGC 7538 IRS9 based on comparison with laboratory spectra (Raunier et al. 2004), but the identification of molecules in the 6-8 $\mu \mathrm{m}$ region is complicated by the overlap of multiple absorption bands, and the results of these two studies are not conclusive (Boogert et al. 2008). What are the reactions that would drive the chemistry of $\mathrm{NH}_{2} \mathrm{CHO}$ in the interstellar medium (ISM)? Although gas phase formation routes to complex molecules exist, it is generally accepted that grain surface chemistry is the dominant formation mechanism for such species (Bisschop et al. 2007a). The $\mathrm{NH}_{2} \mathrm{CHO}$ molecule can form by recombination between the radicals $\mathrm{NH}_{2}$ and $\mathrm{HCO}$ following energetic processing of ices by, for example, UV photons (Allamandola et al. 1999; Muñoz Caro \& Schutte 2003; van Broekhuizen et al. 2004).

It has recently been determined that $\mathrm{NH}_{2} \mathrm{CHO}$ is the most energetically stable $\mathrm{CH}_{3} \mathrm{NO}$ isomer that can be formed, and that the amide bond is the most stable bond possible (Lattelais et al. 2010). Jones et al. (2011) contend that the hydrogenation of HNCO is insignificant as a route to formamide formation because of the low barrier to the reaction $\mathrm{HNCO}+\mathrm{NH}_{3}$ (Raunier et al. 2003a; Mispelaer et al. 2012). While it is true that $\mathrm{NH}_{3}$ does react rapidly with $\mathrm{HNCO}$, the flux of hydrogen atoms onto a grain will be superior to the quantity of $\mathrm{NH}_{3}$ molecules in the ice mantle. This is particularly true before formation of $\mathrm{NH}_{3}$ in the mantle. In order to reproduce observed abundances of HNCO and $\mathrm{OCN}^{-}$in dense molecular clouds, it has been speculated that hydrogenation of HNCO must dominate its destruction mechanisms (Theule et al. 2011). However, our results suggest that hydrogenation is not an efficient process and thus thermal reactions, such as the reaction with $\mathrm{NH}_{3}$ or $\mathrm{H}_{2} \mathrm{O}$, should dominate HNCO grain surface chemistry.

It has already been demonstrated that $\mathrm{CH}_{3} \mathrm{CHO}$ undergoes hydrogenation after $\mathrm{H}$ bombardment, but that it also forms $\mathrm{CH}_{4}$, $\mathrm{H}_{2} \mathrm{CO}$, and $\mathrm{CH}_{3} \mathrm{OH}$ (Bisschop et al. 2007b). The hydrogenation product, ethanol, represents approximately $20 \%$ of the total products formed. The relative strength of the $\mathrm{C}=\mathrm{O}$ bond prevents hydrogenation being the most prevalent reaction, and the C-C bond breaks to form products that are chemically simpler and of lower mass than the original $\mathrm{CH}_{3} \mathrm{CHO}$. Our results confirm that hydrogenation of the $\mathrm{C}=\mathrm{O}$ bond is not favourable under low temperature $\mathrm{H}$ bombardment conditions.

Our results show that the OCN moiety is formed in its radical or anionic form during $\mathrm{H}$ bombardment. The $\mathrm{OCN}^{-}$ion has been positively identified in interstellar ices (Soifer et al. 1979; Grim \& Greenberg 1987) and its rotational spectrum has been measured (Lattanzi et al. 2010), so searches in the gas phase are possible. The neutral OCN (Kawaguchi et al. 1985) has not yet 
been identified in the ISM; it has, however, long been predicted in dense clouds (Prasad \& Huntress 1978). If OCN/OCN ${ }^{-}$can be formed by the reaction of $\mathrm{H}$ with $\mathrm{HNCO}$, this could help explain the destruction of $\mathrm{HNCO}$, while confirming that formamide formation does not occur via this route. Additionally, in dense regions such as pre-stellar cores, H/D exchange is potentially the dominant destruction mechanism for HNCO (Roberts et al. 2003).

We have also shown that formamide does not react with hydrogen to produce aminomethanol, the logical saturated endpoint for HNCO hydrogenation. Aminomethanol has, however, already been shown to form under interstellar conditions by the purely thermal reaction between $\mathrm{H}_{2} \mathrm{CO}$ and $\mathrm{NH}_{3}$, with an activation barrier of $4.5 \pm 0.5 \mathrm{~kJ} \mathrm{~mol}^{-1}(541 \pm 60 \mathrm{~K}$, Bossa et al. 2009). Theoretical studies suggest that, in the presence of acids, aminomethanol can undergo spontaneous dehydration to form methylenimine $\left(\mathrm{CH}_{2}=\mathrm{NH}\right.$ ) and water (Walch et al. 2001); experiments have shown that aminomethanol yields hexamethylenetetramine (HMT) from the polymerisation of the dehydration product methylenimine (Bernstein et al. 1995; Vinogradoff et al. 2011). Although it has not yet been observed in the ISM, in the laboratory HMT has a sublimation temperature of $\sim 553 \mathrm{~K}$ (Bernstein et al. 1995), so it is thought to be present as part of an organic residue on comets. The formation routes to aminomethanol and hydroxyacetonitrile $\left(\mathrm{HOCH}_{2} \mathrm{CN}\right)$ are competitive when $\mathrm{CN}^{-}$is included in the initial ice (Danger et al. 2012). These destruction routes and competitive reactions, combined with our conclusion that aminomethanol does not form by the hydrogenation of formamide, might result in low interstellar abundances of aminomethanol, although it has not yet been observed.

Acknowledgements. This work has been funded by the French national program Physique Chimie du Milieu Interstellaire (PCMI) and the Centre National d'Études Spatiales (CNES). J.A.N. is a Royal Commission for the Exhibition of 1851 Research Fellow.

\section{References}

Acharyya, K., Fuchs, G. W., Fraser, H. J., van Dishoeck, E. F., \& Linnartz, H. 2007, A\&A, 466, 1005

Allamandola, L. J., Bernstein, M. P., Sandford, S. A., \& Walker, R. L. 1999, Space Sci. Rev., 90, 219

Amiaud, L., Fillion, J. H., Baouche, S., et al. 2006, J. Chem. Phys., 124, 094702 Amiaud, L., Dulieu, F., Fillion, J. H., Momeni, A., \& Lemaire, J. L. 2007, J. Chem. Phys., 127, 144709

Atkins, P. A., \& de Paula, J. 2002, Atkins' Physical Chemistry, 7th edn. (Oxford University Press)

Bernstein, M. P., Sandford, S. A., Allamandola, L. J., Chang, S., \& Scharberg, M. A. 1995, ApJ, 454, 327

Bisschop, S. E., Jørgensen, J. K., van Dishoeck, E. F., \& de Wachter, E. B. M. 2007a, A\&A, 465, 913

Bisschop, S. E., Fuchs, G. W., van Dishoeck, E. F., \& Linnartz, H. 2007b, A\&A, 474, 1061

Boogert, A. C. A., Pontoppidan, K. M., Knez, C., et al. 2008, ApJ, 678, 985

Bossa, J. B., Borget, F., Duvernay, F., Theulé, P., \& Chiavassa, T. 2008, J. Phys. Chem. A, 112, 5113

Bossa, J. B., Theule, P., Duvernay, F., \& Chiavassa, T. 2009, ApJ, 707, 1524

Brooke, T. Y., Sellgren, K., \& Geballe, T. R. 1999, ApJ, 517, 883

Brucato, J. R., Baratta, G. A., \& Strazzulla, G. 2006, A\&A, 455, 395

Carter, G. 1962, Vacuum, 12, 245

Celio, H., Mudalige, K., Mills, P., \& Trenary, M. 1997, Surf. Sci., 394, L168

Chaabouni, H., Minissale, M., Manicò, G., et al. 2012, J. Chem. Phys., 137, 234706

Clutter, D. R., \& Thompson, W. E. 1969, J. Chem. Phys., 51, 153

Collings, M. P., Dever, J. W., Fraser, H. J., \& McCoustra, M. R. S. 2003, Ap\&SS, 285,633

Congiu, E., Matar, E., Kristensen, L. E., Dulieu, F., \& Lemaire, J. L. 2009, MNRAS, 397, L96

Congiu, E., Fedoseev, G., Ioppolo, S., et al. 2012, ApJ, 750, L12

Danger, G., Duvernay, F., Theulé, P., Borget, F., \& Chiavassa, T. 2012, ApJ, 756, 11
Demyk, K., Dartois, E., d'Hendecourt, L., et al. 1998, A\&A, 339, 553

Dulieu, F., Amiaud, L., Congiu, E., et al. 2010, A\&A, 512, A30

Dulieu, F., Congiu, E., Noble, J., et al. 2013, Sci. Rep., 3, 1338

Falk, M., \& Giguère, P. A. 1957, Can. J. Chem., 35, 1195

Fedoseev, G., Ioppolo, S., Zhao, D., Lamberts, T., \& Linnartz, H. 2015, MNRAS, 446, 439

Fuchs, G. W., Cuppen, H. M., Ioppolo, S., et al. 2009, A\&A, 505, 629

Garrod, R. T., Weaver, S. L. W., \& Herbst, E. 2008, ApJ, 682, 283

Godfrey, P. D., Brown, R. D., Robinson, B. J., \& Sinclair, M. W. 1973, ApJ, 13, L119

Grim, R. J. A., \& Greenberg, J. M. 1987, ApJ, 321, L91

Hamada, Y., Hashiguchi, K., Tsuboi, M., Koga, Y., \& Kondo, S. 1984, J. Mol. Spectr., 105, 70

Hasegawa, T. I., Herbst, E., \& Leung, C. M. 1992, ApJS, 82, 167

Helmich, F. P., \& van Dishoeck, E. F. 1997, A\&AS, 124, 205

Hiraoka, K., Ohashi, N., Kihara, Y., et al. 1994, Chem. Phys. Lett., 229, 408

Hiraoka, K., Miyagoshi, T., Takayama, T., Yamamoto, K., \& Kihara, Y. 1998, ApJ, 498, 710

Iglesias, E. 1977, ApJ, 218, 697

Ioppolo, S., Cuppen, H. M., Romanzin, C., van Dishoeck, E. F., \& Linnartz, H. 2008, ApJ, 686, 1474

Ioppolo, S., van Boheemen, Y., Cuppen, H. M., van Dishoeck, E. F., \& Linnartz, H. 2011, MNRAS, 413, 2281

Jackson, J. M., Armstrong, J. T., \& Barrett, A. H. 1984, ApJ, 280, 608

Jones, J. E., \& Trenary, M. 2008, J. Phys. Chem. C, 112, 20443

Jones, B. M., Bennett, C. J., \& Kaiser, R. I. 2011, ApJ, 734, 78

Kaifu, N., Morimoto, M., Nagane, K., et al. 1974, ApJ, 191, L135

Kawaguchi, K., Saito, S., \& Hirota, E. 1985, Mol. Phys., 55, 341

Keane, J. V., Tielens, A. G. G. M., Boogert, A. C. A., Schutte, W. A., \& Whittet, D. C. B. 2001, A\&A, 376, 254

King, D. A. 1975, Surf. Sci., 47, 384

Kiss, J., \& Solymosi, F. 1983, Surf. Sci., 135, 243

Kristensen, L. E., Amiaud, L., Fillion, J.-H., Dulieu, F., \& Lemaire, J.-L. 2011, A\&A, 527, A44

Lacy, J. H., Baas, F., Allamandola, L. J., et al. 1984, ApJ, 276, 533

Lattanzi, V., Gottlieb, C. A., Thaddeus, P., Thorwirth, S., \& McCarthy, M. C. 2010, ApJ, 720, 1717

Lattelais, M., Pauzat, F., Ellinger, Y., \& Ceccarelli, C. 2010, A\&A, 519, A30

Li, D., \& Goldsmith, P. F. 2003, ApJ, 585, 823

Lis, D. C., Keene, J., Young, K., et al. 1997, Icarus, 130, 355

Lowenthal, M. S., Khanna, R. K., \& Moore, M. H. 2002, Spectrochim. Acta Part A, 58, 73

Minissale, M., Congiu, E., \& Dulieu, F. 2014, J. Chem. Phys., 140, 074705

Mispelaer, F., Theule, P., Duvernay, F., Roubin, P., \& Chiavassa, T. 2012, A\&A, 540, A40

Miyauchi, N., Hidaka, H., Chigai, T., et al. 2008, Chem. Phys. Lett., 456, 27

Mokrane, H., Chaabouni, H., Accolla, M., et al. 2009, ApJ, 705, L195

Moore, M. H., Donn, B., Khanna, R., \& A'Hearn, M. F. 1983, Icarus, 54, 388

Muñoz Caro, G. M., \& Schutte, W. A. 2003, A\&A, 412, 121

Németh, R., Kiss, J., \& Solymosi, F. 2007, J. Phys. Chem. C, 111, 1424

Nguyen-Q-Rieu, Henkel, C., Jackson, J. M., \& Mauersberger, R. 1991, A\&A, 241, L33

Nguyen, M. T., Sengupta, D., Vereecken, L., Peeters, J., \& Vanquickenborne, L. G. 1996, J. Phys. Chem., 100, 1615

Noble, J. A., Dulieu, F., Congiu, E., \& Fraser, H. J. 2011, ApJ, 735, 121

Noble, J. A., Congiu, E., Dulieu, F., \& Fraser, H. J. 2012, MNRAS, 421, 768

Noble, J. A., Theule, P., Borget, F., et al. 2013, MNRAS, 428, 3262

Prasad, S. S., \& Huntress, W. T., Jr. 1978, MNRAS, 185, 741

Raunier, S., Chiavassa, T., Marinelli, F., Allouche, A., \& Aycard, J.-P. 2003a, Chem. Phys. Lett., 368, 594

Raunier, S., Chiavassa, T., Allouche, A., Marinelli, F., \& Aycard, J.-P. 2003b, Chem. Phys., 288, 197

Raunier, S., Chiavassa, T., Duvernay, F., et al. 2004, A\&A, 416, 165

Redhead, P. A. 1962, Vacuum, 12, 203

Roberts, H., Herbst, E., \& Millar, T. J. 2003, ApJ, 591, L41

Rodríguez-Fernández, N. J., Tafalla, M., Gueth, F., \& Bachiller, R. 2010, A\&A, 516, A98

Rubin, R. H., Swenson, G. W., Jr., Benson, R. C., Tigelaar, H. L., \& Flygare, W. H. 1971, ApJ, 169, L39

Saladino, R., Botta, G., Pino, S., Costanzo, G., \& Di Mauro, E. 2012, Chem. Soc. Rev., 41, 5526

Schutte, W. A., \& Khanna, R. K. 2003, A\&A, 398, 1049

Schutte, W. A., Gerakines, P. A., Geballe, T. R., van Dishoeck, E. F., \& Greenberg, J. M. 1996, A\&A, 309, 633

Schutte, W. A., Boogert, A. C. A., Tielens, A. G. G. M., et al. 1999, A\&A, 343, 966

Snyder, L. E., \& Buhl, D. 1972, ApJ, 177, 619 


\section{J. A. Noble et al.: Hydrogenation of HNCO}

Snyder, L. E., Buhl, D., Zuckerman, B., \& Palmer, P. 1969, Phys. Rev. Lett., 22, 679

Soifer, B. T., Puetter, R. C., Russell, R. W., et al. 1979, ApJ, 232, L53

Theule, P., Borget, F., Mispelaer, F., et al. 2011a, A\&A, 534, A64

Theule, P., Duvernay, F., Ilmane, A., Coussan, S., \& Chiavassa, T. 2011b, A\&A, 530, A96

Tideswell, D. M., Fuller, G. A., Millar, T. J., \& Markwick, A. J. 2010, A\&A, 510, A85

Tielens, A. G. G. M., \& Hagen, W. 1982, A\&A, 114, 245

van Broekhuizen, F. A., Keane, J. V., \& Schutte, W. A. 2004, A\&A, 415, 425 van Broekhuizen, F. A., Pontoppidan, K. M., Fraser, H. J., \& van Dishoeck, E. F. 2005, A\&A, 441, 249

Vasyunina, T., Linz, H., Henning, T., et al. 2011, A\&A, 527, A88

Vinogradoff, V., Duvernay, F., Danger, G., Theulé, P., \& Chiavassa, T. 2011, A\&A, 530, A128

Walch, S., Bauschlicher, C., Ricca, A., \& Bakes, E. 2001, Chem. Phys. Lett., 333,6

Watanabe, N., \& Kouchi, A. 2002, ApJ, 571, L173

Wilson, R. W., Jefferts, K. B., \& Penzias, A. A. 1970, ApJ, 161, L43

Zinchenko, I., Henkel, C., \& Mao, R. Q. 2000, A\&A, 361, 1079 